\title{
Periprosthetic fractures of the femur: the stability of the implant dictates the type of treatment
}

\author{
Francesco Biggi - Stefano Di Fabio • \\ Corrado D'Antimo $\cdot$ Silvia Trevisani
}

Published online: 9 March 2010

(C) Springer-Verlag 2010

The increasing incidence of complications after hip replacement surgery is a consequence of two main factors: the prolonged survival time in the general population and the increasing number of primary implants made worldwide in the last decades. Among the possible complications, periprosthetic femoral fractures represent a challenge for orthopaedic surgeons. In terms of treatment, it is crucial to distinguish between intraoperative (or perioperative) fractures and post-traumatic fractures. The former may be minor fractures that do not necessitate surgical treatment. Posttraumatic fractures usually require operative treatment and are associated both with predisposing factors such as osteolysis, osteopenia and aseptic loosening of the implant as well as with determining factors such as minor traumatic events.

The incidence of intraoperative periprosthetic fractures in cementless total hip arthroplasty ranges from 1 to $20 \%$ and is higher in revision surgery $[2,6,7,8,11,15]$. Lower rates are observed in cemented procedures where the need for a tight press fit is less. These fractures are usually located in the calcar region or directly in the trochanter, and they are often the consequence of excessive reaming with rasps and broaches during preparation of the medullary canal or, inadvertently, during insertion of the definitive stem. Although fixation of intraoperative fractures with wire cerclage or plates is effective, it is recommended to avoid such events through careful preoperative planning and optimal surgical technique.

Regarding postoperative periprosthetic fractures, the incidence ranges between 1 and 4\%, again with higher rates in revision surgery $[2,6,10,12]$. These fractures are

F. Biggi · S. Di Fabio $(\bowtie) \cdot$ C. D’Antimo $\cdot$ S. Trevisani Orthopaedics and Traumatology Department,

San Martino Hospital, Viale Europa 22, 32100 Belluno, Italy

e-mail: stdifabio74@hotmail.com sometimes considered pathological because the causative factors, besides minor trauma, are aseptic loosening, primary and secondary osteoporosis, and conditions predisposing to osteopenia (e.g. rheumatoid arthritis, Paget's disease, Parkinson's disease, poliomyelitis, myasthenia gravis and polyneuropathies).

The Vancouver classification of periprosthetic fractures of the hip $[4,5]$ is considered a reliable system for grading these fractures as well as for guiding treatment decisions. In particular, the Vancouver classification helps distinguish stable from unstable fractures requiring fixation as well as stable from unstable implants requiring revision. Fractures involving the trochanteric area are categorized as type A (Ag and $\mathrm{Al}$ for the greater and lesser trochanter, respectively), fractures about the stem or tip of the implant are type $B$, and fractures distal to the tip of the stem are type $C$. Type B fractures are further divided into subtype B1 when adjacent to a well fixed stem, B2 in presence of a loose stem, and B3 when associated with marked osteopenia or loss of bone substance. According to this classification system, most of these fractures require surgical treatment.

The choice of treatment is based upon the type of fracture, the integrity and quality of the bone stock, and the stability of the original implant according to an algorithm proposed by Masri et al. [13, 18]. Sometimes in selected type A fractures, it is helpful to test the stability with stress manoeuvres performed under fluoroscopic visualization. However, conservative treatment in non-selected cases usually requires prolonged periods of bed rest (with consequential functional disability), which is not recommended for elderly persons who often have other diseases and poor general conditions.

Type A fractures, created by a simple avulsion of the greater or lesser trochanter, are usually treated conservatively with bed and chair rest for 3-4 weeks and have good 
Fig. 1 Antero-posterior (a) and axial (b) view of a type A1 fracture in a 68 year old woman treated with internal fixation by GTR plate (Zimmer, Warsaw, USA)
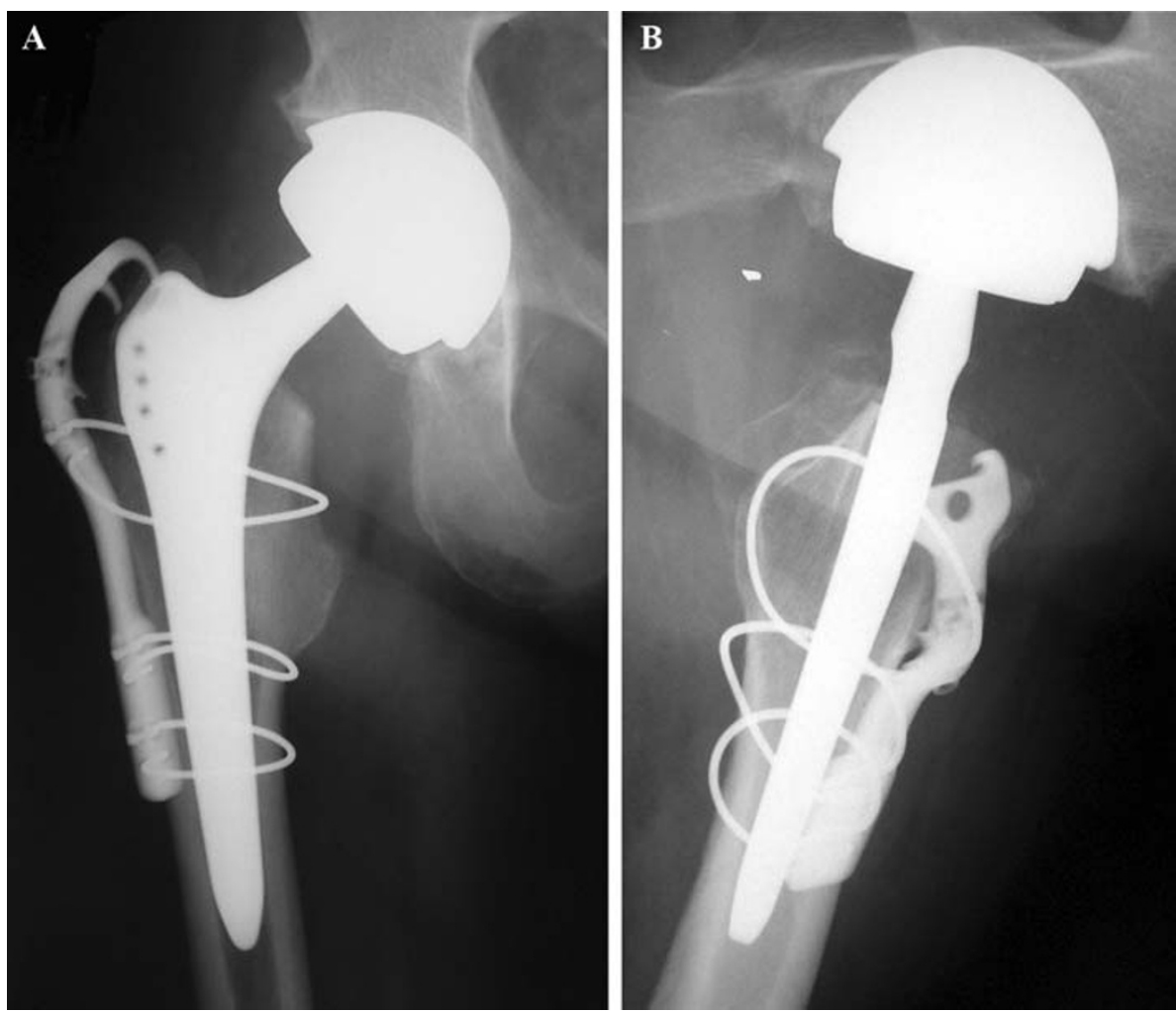

functional outcomes. In case of displacement of the greater trochanter, the general consensus is to fix it using cerclage wires or plates, in order to restore the functional leverage of the glutei muscles (Fig. 1a, b).

Type B1 fractures are, by definition, associated with a well fixed stem and can be treated successfully with open reduction and internal fixation (ORIF). For this purpose, plates specifically contoured to the trochanteric area are useful, because they can be fixed with cortical screws or cerclage wires (Figs. 2, 3a, b). In the presence of local osteolysis, it is often necessary to improve implant stability by applying a cortical structural allograft. Biomechanical studies [1, 3, 9, $14,16,17]$ have shown that the strongest configuration system is achieved with either a trochanteric plate with proximal unicortical and distal bicortical screws or a plate with proximal unicortical screws combined with cerclage wires and distal bicortical screws. The healing rate for type B1 fractures, treated with these techniques, is above $90 \%$. Failure has been reported in association with a varus deformity of the stem or insufficient osteointegration of the implant.

Type B2 fractures, and all other fractures associated with a loose femoral component, are best treated with implant revision (Figs. 4, 5a, b). By using non-cemented modular stems, it is possible to bypass the fracture site and achieve distal cortical fixation for at least $7 \mathrm{~cm}$ (a distance twice that of the femoral canal diameter). With this construct in place, final reconstruction of the proximal femur around the stem is easier to achieve.

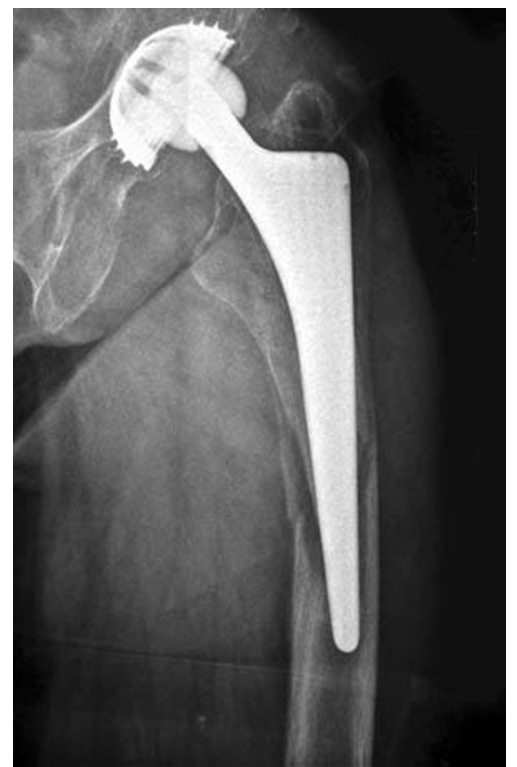

Fig. 2 A type B1 fracture in a 71 year old woman

In type B3 fractures, simple revision surgery is often inadequate for healing. In fact, due to the great amount of bone loss, it is advisable to augment the revision surgery with cancellous bone impaction grafting or strut-grafting with cerclage wires, in order to enhance the stability of the entire construct (Figs. 6, 7a, b).

Type $\mathrm{C}$ fractures are preferably treated with ORIF, through the use of plates with screws and cerclage wires. 
Fig. 3 Postoperative

radiograph following open

reduction and internal fixation

with GTR plate
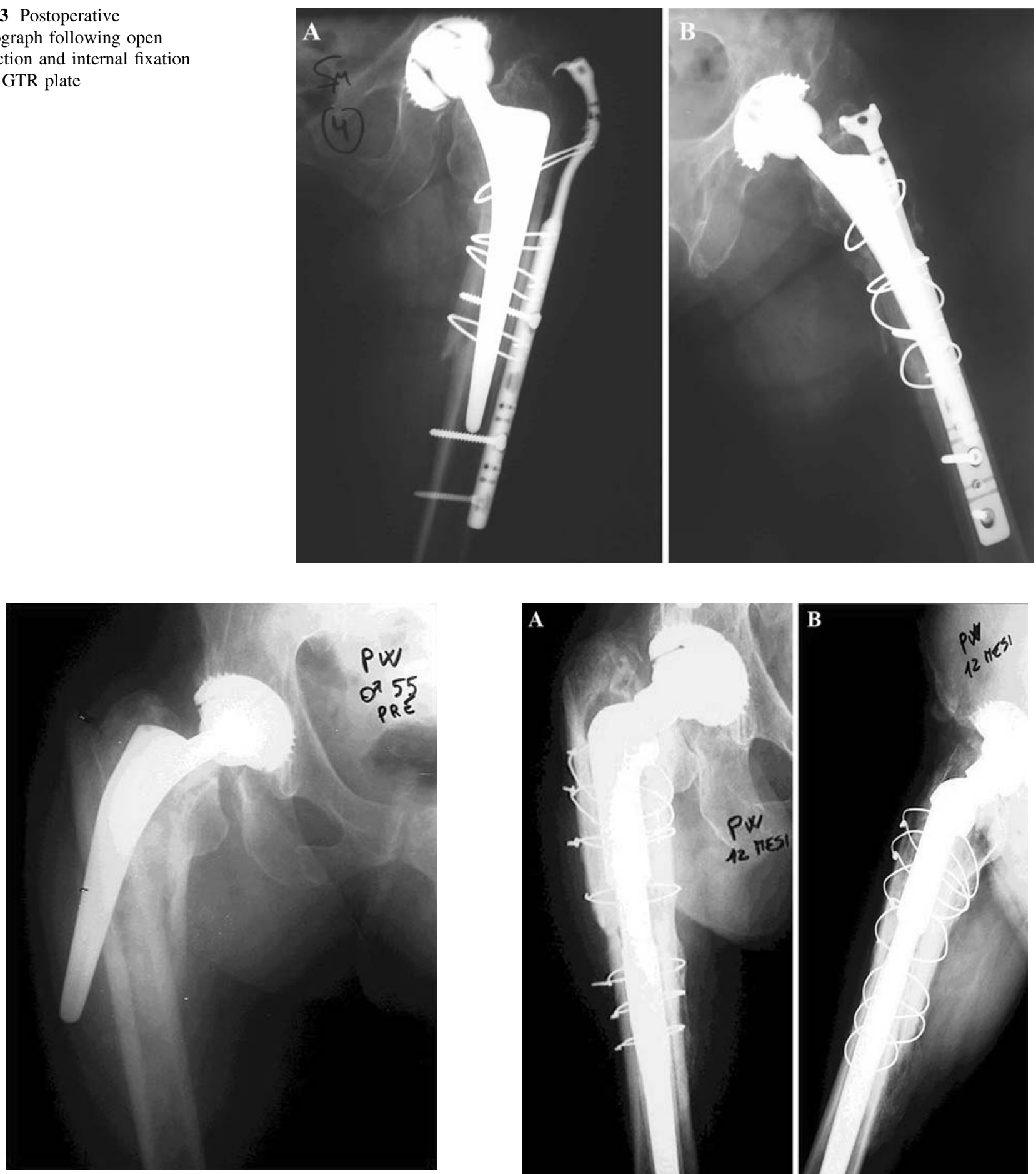

Fig. 4 A type B2 fracture in a 78 year old man

These devices allow good primary stability of the implant, early recovery of weight bearing, and good final functional outcomes. Alternatively, it is possible to use retrograde femoral nailing for very distal fractures of the femur, located more than $6 \mathrm{~cm}$ from the tip of the stem.

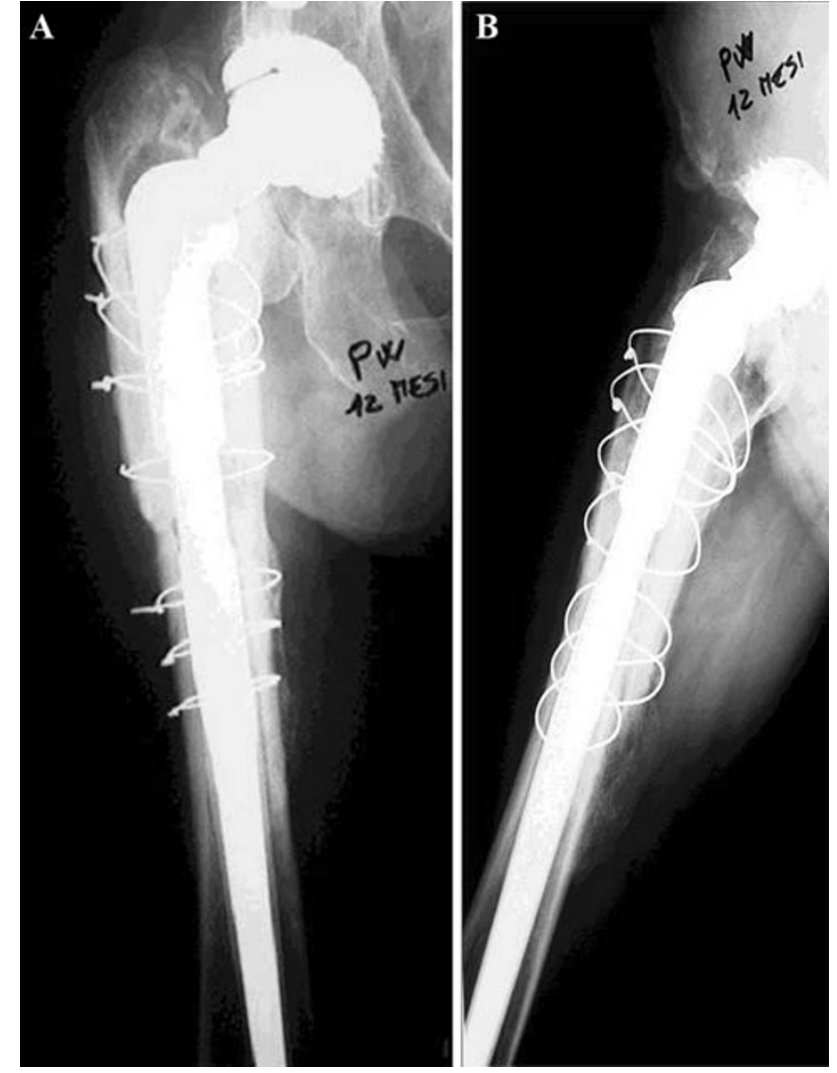

Fig. 5 The Anteroposterior (a) and lateral (b) radiographs after struction hip stem, Waldemar Link, Hamburg, Germany) replacement of the proximal femur with a long stem (MP recon- 


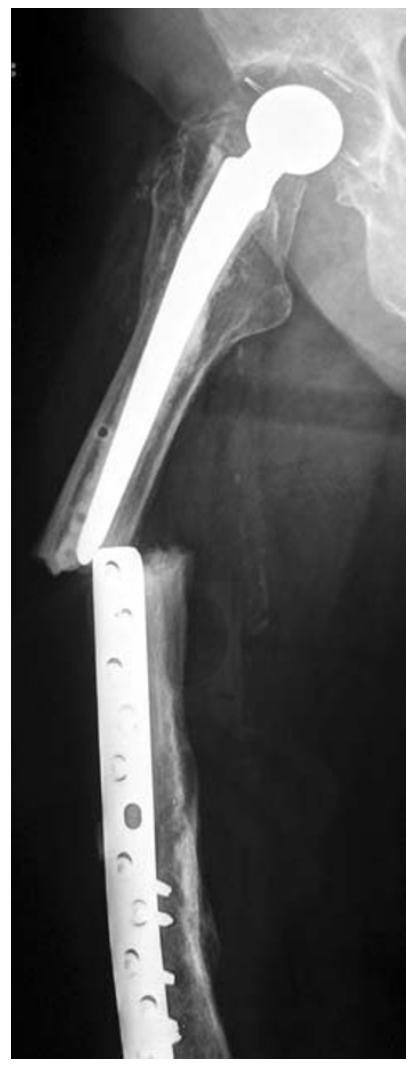

Fig. 6 A type B3 fracture in a 83 year old woman

The current gold standard for the treatment of posttraumatic periprosthetic femoral fractures is surgery, with an exception for selected simple fractures having a stable implant, which can be treated conservatively with bed rest, traction, casts or braces. Consequently, it is imperative to correctly identify the type of fracture and the stability of the implant for correct surgical planning. The Vancouver system is helpful to guide treatment choices, although the most reliable way to ascertain stability of the femoral stem is by intraoperative evaluation. When ORIF is indicated, the use of plates, proximally hooked in an anatomical configuration to the greater trochanter and accepting screws, cerclage wires or cables for transcortical fixation, are of great utility. Structural cortical auto- and allografts are also indicated to augment the fixation in cases of severe comminution or insufficient cortical bone stock. In selected fractures adjacent to an unstable stem, implant revision is mandatory. For this purpose, we prefer to use a noncemented, modular long stem, with distal cortical fixation and antirotational slots, which allows us to reconstruct the proximal femur around the stem. This option is helpful when the proximal femur is comminuted from a traumatic event or osteotomized for the revision of a previously inserted cemented stem. Furthermore, the modularity of this implant allows us to adopt any last minute changes to
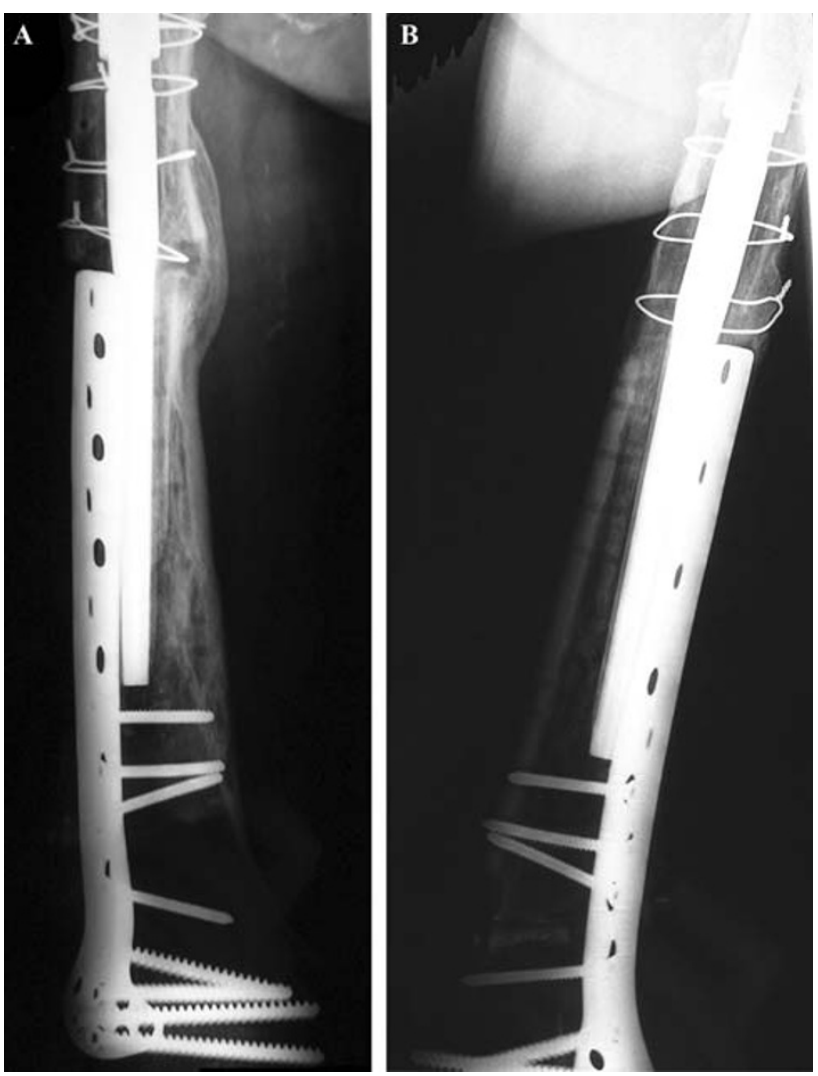

Fig. 7 The anteroposterior (a) and lateral (b) radiographs after replacement of the proximal femur with a long stem (MP reconstruction hip stem) and fixation with four cables and bone graft

correct leg length discrepancies, and to achieve the necessary articular stability by balancing the soft tissues. However, irrespective of the surgical treatment adopted, it is of paramount importance to understand that the final result also depends on early functional recovery and social independence, achieved only with an effective rehabilitation program and social support. Finally, notwithstanding the technical aspects of the treatment of these fractures, we should always inform patients about the real expectations from these difficult clinical situations.

Conflict of interest statement None.

\section{References}

1. Beals RK, Tower SS (1996) Periprosthetic fractures of the femur. An analysis of 93 fractures. Clin Orthop Relat Res 327:238-246

2. Berry DJ (1999) Epidemiology of periprosthetic fractures after major joint replacement: hip and knee. Orthop Clin North Am 30:183-190

3. Bethea JS III, De Andrade JR, Fleming LL, Lindenbaum SD, Welch RB (1982) Proximal femoral fractures following total hip arthroplasty. Clin Orthop Relat Res 170:95-106 
4. Brady OH, Garbuz DS, Marsi BA, Duncan CP (1999) Classification of the hip. Orthop Clin North Am 30:215-220

5. Brady OH, Garbuz DS, Marsi BA, Duncan CP (2000) The reliability and validity of the Vancouver classification of femoral fractures after hip replacement. J Arthroplasty 15:59-62

6. Duncan CP, Masri BA (1995) Fractures of the femur after hip replacement. Instr Course Lect 44:293-304

7. Fitzgerald RH Jr, Brindley GW, Kavanagh BF (1988) The uncemented total hip arthroplasty: intraoperative femoral fractures. Clin Orthop Relat Res 235:61-66

8. Giannini S, Moroni A, Piras F, Guzzardella M, Faldini C, Mosca M (1997) Epidemiologia delle fratture di femore nelle artroprotesi d'anca. Giorn Ital Ortop Traum Suppl XXIII(3):19-28

9. Haddad FS, Duncan CP, Berry DJ, Lewallen DG, Gross AE, Chandler HP (2002) Periprosthetic femoral fractures around wellfixed implant: use of cortical onlay allografts with or without a plate. J Bone Joint Surg Am 84:945-950

10. Kavanagh BF (1992) Femoral fractures associated with total hip arthroplasty. Orthop Clin North Am 23:249-257
11. Lee SR, Bostrom MP (2006) Periprosthetic fractures of the femur after total hip arthroplasty. Instr Course Lect 19:253-260

12. Lewallen DJ, Berry DJ (1998) Periprosthetic fracture of the femur after total hip arthroplasty: treatment and results to date. Instr Course Lect 47:243-249

13. Masri BA, Meek RM, Duncan CP (2004) Periprosthetic fractures evaluation and treatment. Clin Orthop Relat Res 420:80-95

14. Rosemberg AG (2006) Managing periprosthetic femoral stem fractures. J Arthroplasty 21:101-104

15. Schmidt AH, Kyle RF (2002) Periprosthetic fractures of the femur. Orthop Clin N Am 33:143-152

16. Tadross TS, Nanu AM, Buchanan MJ, Checketts RG (2000) Dall-Miles plating for periprosthetic B1 fractures of the femur. J Arthroplasty 15:47-51

17. Tsiridis E, Krikler S, Giannoudis PV (2007) Periprosthetic femoral fractures: current aspect of management. Injury 38(6):649650

18. Wilson D, Masri BA, Duncan CP (2001) Periprosthetic fractures: an operative algorithm. Orthopedics 24:869-870 